\title{
The Evolution of Low Mass Contact Binaries
}

\author{
Kazimierz Stępien ${ }^{1}$ and Kosmas Gazeas ${ }^{2,3}$ \\ ${ }^{1}$ Warsaw University Observatory, Al. Ujazdowskie 4, 00-478 Warszawa, Poland \\ email: kst@astrouw.edu.pl \\ ${ }^{2}$ Department of Astrophysics, Astronomy and Mechanics, Faculty of Physics, University of \\ Athens, GR-157 84, Zografos, Athens, Greece \\ ${ }^{3}$ European Space Agency, ESTEC, Mechatronics and Optics Division, Keplerlaan 1, 2200AG, \\ Noordwijk, The Netherlands \\ email: kgaze@physics.auth.gr, Kosmas.Gazeas@esa.int
}

\begin{abstract}
We discuss the origin and evolution of low mass contact binaries with $P_{\text {orb }}$ shorter than $0.3 \mathrm{~d}$ that have properties somewhat different from the rest of the contact binaries. A comparison of an evolutionary model set with observations shows that both components are on the main sequence, the age of the binaries is at least several Gyr, while the contact phase lasts only less than 1 Gyr.
\end{abstract}

Keywords. contact binaries, W UMa stars, stellar evolution

\section{Evolution of contact binaries, and the suggested new model}

The problem of the origin and evolution of cool contact binaries is still far from full understanding. The TRO theory explains the situation quite well. However, it encounters several problems and some of its predictions are at odds with observations (Webbink 2003, Stȩpień 2011). A different theory has been suggested recently (Stȩpień 2006, 2009, Gazeas \& Stȩpień 2008), which assumes that mass transfer following Roche lobe overflow (RLOF) proceeds in a similar way as in classical Algols, i.e. until mass ratio reversal and angular momentum (AM) loss, which lead to a contact configuration.

The new model is based on the assumption that the initial $P_{\text {orb }}$ of progenitors of cool contact binaries is close to a couple of days. It takes several Gyr until enough AM is lost and RLOF occurs. This time is sufficient to terminate the main sequence (MS) evolution, having components with mass higher than 1 solar mass. After mass exchange, the low mass components or LMCs (former high mass components or HMCs) of such binaries have hydrogen depleted cores. A new set of models of LMCBs was calculated by Stȩpień (2011), The initial parameters of these models are: $0.9+0.3(2.5), 0.9+0.4(2.5)$, $0.9+0.5(2.0), 0.9+0.5(1.5), 0.9+0.7(2.0), 1.1+0.5(2.0)$ and $1.1+0.5(1.5)$, where the first two numbers give the initial component masses in solar units and the number in parentheses gives the initial $P_{\text {orb }}$ in days. It takes, on average, about 7 Gyr for each binary to reach the contact phase, but they live in a contact configuration for about 0.8 Gyr. After this phase, the orbit is so tight, that both stars overflow their outer critical surfaces. The binary loses mass and AM through the $L_{2}$ point, which results in its coalescence. Both components are still on the MS, although the LMC may be close to (or slightly beyond) the terminal age MS (TAMS) region.

Observations show that there are several low mass contact binaries (LMCB) with the total mass close to 1-1.4 $M_{\odot}$ (Gazeas \& Niarchos 2006, Gazeas \& Stẹpień 2008). The LMCB compared with our models include the first 9 stars from Table 1 of Gazeas \& Stȩpień (2008). These systems have $P_{\text {orb }}$ shorter than $0.3 \mathrm{~d}$ and their orbital AM is low (significantly less than $3 \times 10^{51}$ in cgs units), while the component masses and 
radii indicate that they are both on the MS (Fig. 1). The HMC in all LMCB appear to have lower average surface brightness than their lower mass companions, which classifies them as W-type systems, according to the classification made by Binnendijk (1970). The heavily covered surface of the HMC by cool spots is the most likely explanation of the so-called $W$ - phenomenon (Stȩpień 1980, Eaton, Wu \& Rucinski 1980, Zola et al. 2010).
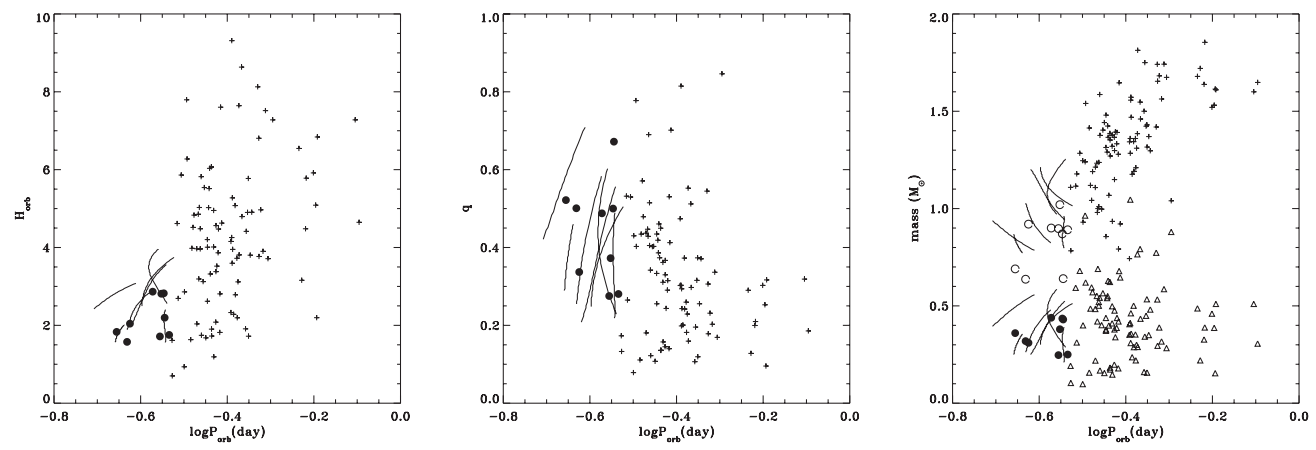

Figure 1. The plots present the comparison of the computed models with the observations for the $H_{o r b}$ vs $P_{o r b}$ (left) and the mass ratio vs $P_{\text {orb }}$ (center) of massive contact binaries (crosses) and LMCB (filled circles). Each model (solid line) corresponds to the time evolution of one of the seven model binaries, from the time when the components reach contact till they both overflow the outer critical surface, which results in their quick merging (Webbink 1977). Direction of evolution is from higher to lower values of $H_{\text {orb }}$ and the same for $q$. Separate component masses of LMCB are shown in right panel. Here, crosses and triangles correspond, respectively, to HMC and LMC of massive CBs, whereas open and filled circles correspond to the same components of LMCB. The solid lines show evolution of the component masses from right to left.

\section{Conclusions}

Low-mass contact binaries have $P_{\text {orb }}$ shorter than $0.3 \mathrm{~d}$, total mass lower than about $1.4 M_{\odot}$, orbital AM less than about $3 \times 10^{51}$ (in cgs units), radii corresponding to MS stars, relatively high mass ratios, and none of them is of A-type. According to our models, they originate from detached binaries with total initial mass lower than 1.6 solar mass and initial $P_{\text {orb }}$ of $1.5-2 \mathrm{~d}$. Evolution is driven mostly by mass transfer and AM loss via the magnetized wind, which shrinks the orbit and makes both components overflow their outer Roche lobes. All LMCB are old, with a typical age of 7-8 Gyr, although their contact phase lasts less than 1 Gyr, leading into coalescence.

\section{References}

Binnendijk, L., 1970, Vistas in Astron., 12, 217

Eaton, J., Wu, C. C., \& Rucinski, S. M., 1980, ApJ, 239, 919

Gazeas, K. D. \& Niarchos, P. G., 2006, MNRAS, 370, L29

Gazeas, K. \& Stępień, K., 2008, MNRAS, 390, 1577

Stępień, K., 1980, AcA, 30, 315

Stȩpień, K., 2006, AcA, 56, 199

Stȩpień, K., 2009, MNRAS, 397, 857,

Stępień, K., 2011, AcA, 61, in press

Webbink, R. F., 1977, ApJ, 211, 881

Webbink, R. F., 2003, in: 3D Stellar Evolution, ASP-CS, Vol. 293, S. Turcotte et al. (eds), p. 76

Zola, S., Gazeas, K., Kreiner, J. M., et al. 2010, MNRAS, 408, 474 